\title{
CHARGING MECHANISMS FOR PARTICLES PRIOR. TO ELECTROSTATIC SEPARATION
}

\author{
K.S. LINDLEY AND N.A. ROWSON \\ University of Birmingham, School of Chemical Engineering, Edgbaston, \\ Birmingham B15 2TT, England
}

(Received May 14, 1996, accepted July 8, 1996)

\begin{abstract}
The four principal methods available for charging particles prior to electrostatic separation are by grinding, triboelectrification, corona charging and charging by induction. Each of these mechanisms gives rise to surface charge and are discussed in this paper. Each is a distinct mechanism but in most practical separation processes two or more will occur.
\end{abstract}

\section{CHARGING BY GRINDING}

For a crystal without cleavage planes, for example quartz, comminution produces charged fragments. A positive charge is linked with the electropositive atom (silicon) and a negative charge is paired to the electronegative atom (oxygen) for each rupture site. This leads to a surface with a continuous array of charges. As the time between fractures is very small, of the order of $10^{-13}$ seconds, the material being comminuted lacks time to adjust the charge at the place of fracture and so the sign of charge at contiguous sites is often non-uniform.

On the assumption of total randomness, particles of a single crystal fragment offer $2 n$ surface sites, nominally in $n$ positive sites and $n$ negative sites. According to the binomial theorem and Bernoulli theorem, the number of positive sites will differ on each particle from the number of negative sites by a quantity $r$ : 


$$
\mathrm{r}=\mathrm{x} \sqrt{\mathrm{n}}
$$

and the probability $y$ of this difference being related to $x$ is given by:

$$
y=\frac{2}{\sqrt{\pi}} \int_{0}^{x} e^{-x^{2}} d x
$$

If the material being broken has natural cleavage planes, then each face of the cleavage may have an even or an odd number of ions, this is highly dependent on the linear geometry. Each face can be regarded equivalent to each site for the case of solids without cleavage. There are likely to be more sites than faces so a material possessing a cleavage should have less charge, on average, than one without cleavage [1].

Quartz is normally crushed in moist air which leads to water molecules adsorbing on the fracture surfaces. These water molecules ionise to $\mathrm{H}^{+}$and $\mathrm{OH}^{-}$ions and, as this process occurs in pairs, any excess charges on the fragments are unaffected. Any free ions present in air will reduce the charges gradually.

Any deviation from an ideal crystal structure, for example the inclusion of impurities, will tend to occur at fracture points as cracks propagation is likely here. This may result in a different net charge than that of a pure crystal.

For particles of the order $1 \mathrm{~mm}$, this charge will be insufficient to influence motion in an electric field. For fine particles, the charge-to-weight ratio is high and they will be affected by an electric field [2].

TRIBOELECTRIFICATION (Charging dissimilar particles by friction)

Contact electrification can occur at solid-solid, liquid-liquid or solid-liquid interfaces. For dissimilar solids, initially uncharged and normally at earth potential, a small amount of charge is transferred from one material to the other when they make contact. The two materials become oppositely charged, the 
surfaces acquire a net electric charge, and as a result an electric field exists between them [3].

If one or both materials are non-conductors, the recombination of charge cannot take place completely and the separating materials retain their charge. Repeated contacting is required to build up an appreciable charge due to the small area of contact. If the materials are insulators, then the charge density at the surface can become sufficient to enable separation because charge dissipation is slow. This method of charging is commonly referred to as triboelectrification or frictional charging $[2,4,5]$.

None of the literature on this subject discusses the exact mechanism of charge transfer. It is thought that it is predominantly due to electron transfer, although ion transfer is possible for some materials. Several charge transfer mechanisms have been suggested, electron or ion transfer by surface properties, electron or ion transfer by bulk properties, or transfer related to mechanical dislodgement. The polarity and magnitude of charge could be influenced by many variables including humidity, temperature, dust, gaseous pollutants, external electric fields etc. Additional effects of rubbing, for instance transient high temperature spots, surface damage or alteration, are far from being understood [6].

Coehn's rule states that when two materials are contacted and separated, the material with the higher dielectric constant becomes positively charged. Coehn's rule can be expressed quantitatively as:

$$
\frac{\mathrm{Q}}{\mathrm{A}}=15 \times 10^{-6}\left(\mathrm{e}_{\mathrm{r} 1}-\mathrm{e}_{\mathrm{r} 2}\right)
$$

where $Q / A$ is the surface charge density $\left[\mathrm{Cm}^{-2}\right], \mathrm{e}_{\mathrm{r} 1}$ and $\mathrm{e}_{\mathrm{r} 2}$ are the dielectric constants of the two materials [7].

Materials may be arranged in a table according to the amount of positive charge which is transferred (i.e. every material charges positive against materials below it in the table). This is known as the triboelectric series and is analogous to the electrochemical series of metals. See Table 1 for an early example of a triboelectrification series [8]. This rule is unreliable due to the anomalies which occur with changes in atmospheric conditions and trace impurities in the materials and no two triboelectrification series have been identical $[7,9]$. 
Table 1 An early example of a frictional electricity series (also referred to as the triboelectrification series) [8]

\section{Positive end of the series}

\section{SUBSTANCE}

Serpentine

Asbestos

Fur

Topaz

Mica

Glass

Calcite

Aragonite

Cheesecloth

Barite

Quartz

Magnesium

Fluorite

Lead

Gypsum

Celestine

Zinc

Orthoclase

Anhydrite

Beryl

Blotting paper

Sealing wax

Sphalerite

Magnetite

Galena

Pyrite

Molybdenite

Copper

Antimony

Stibnite

Silver

Silicon

Sulphur

Rubber

\section{COMPOSITION}

$\mathrm{H}_{4} \mathrm{Mg}_{3} \mathrm{Si}_{2} \mathrm{O}_{9}$

Silicate

Organic

$(\mathrm{AlOH})_{2} \mathrm{SiO}_{4}$

$\mathrm{KH}_{2} \mathrm{Al}_{3}\left(\mathrm{SiO}_{4}\right)_{3}$

Silicate

$\mathrm{CaCO}_{3}$

$\mathrm{CaCO}_{3}$

Organic

$\mathrm{BaSO}_{4}$

$\mathrm{SiO}_{2}$

$\mathrm{Mg}$

$\mathrm{CaF}_{2}$

$\mathrm{Pb}$

$\mathrm{CaSO}_{4} \cdot 2 \mathrm{H}_{2} \mathrm{O}$

$\mathrm{SrSO}_{4}$

$\mathrm{Zn}$

$\mathrm{KAlSi}_{3} \mathrm{O}_{8}$

$\mathrm{CaSO}_{4}$

$\mathrm{Be}_{3} \mathrm{Al}_{2} \mathrm{Si}_{6} \mathrm{O}_{18}$

Organic

Organic

$\mathrm{ZnS}$

$\mathrm{Fe}_{3} \mathrm{O}_{4}$

$\mathrm{PbS}$

$\mathrm{FeS}_{2}$

$\mathrm{MoS}_{2}$

$\mathrm{Cu}$

$\mathrm{Sb}$

$\mathrm{Sb}_{2} \mathrm{~S}_{3}$

$\mathrm{Ag}$

$\mathrm{Si}$

$S$

Organic

\section{CRYSTALLISATION}

Monoclinic

Rhombic

Rhombic

Hexagonal

Rhombic

Rhombic

Hexagonal

Isometric

Isometric

Monoclinic

Rhombic

Isometric

Monoclinic

Rhombic

Hexagonal

Isometric

Isometric

Isometric

Isometric

Hexagonal

Isometric

Hexagonal

Rhombic

Rhombic

Negative end of the series 
If a mechanical method enables regular contact in order to accumulate sufficient surface charge, then the charging rate has been shown to depend on:

- $\quad$ surface area of particles being contacted

- frequency of particle collision

- conductivity of the mineral species.

Triboelectrification involves two phenomena:

1. Backflow of electronic charge which occures when two material;s are separated

2. Electronic charge transfer across the interface at the contact point of the two solids.

Hence the charging rate can be considered in terms of the addition of new charge and the loss of existing charge:

$$
\frac{\mathrm{dQ}}{\mathrm{dN}}=\mathrm{Q}_{\mathrm{c}}\left[1-\exp \left(-\mathrm{t}_{\mathrm{b}} / \tau_{\mathrm{b}}\right)\right]-\mathrm{Q}\left[1-\exp \left(-\mathrm{t}_{\mathrm{c}} / \tau_{\mathrm{c}}\right)\right]
$$

where $Q$ is the total charge [C], $N$ is the number of contacts, $t_{\mathrm{b}}$ refers to the time between contacts $[\mathrm{s}], t_{\mathrm{c}}$ refers to the time of contact $[\mathrm{s}], Q_{\mathrm{c}}$ is the charge in the region of contact during contact time $t_{\mathrm{b}}[\mathrm{C}], \tau_{\mathrm{b}}$ represents the time constant for the initial decay of charge $[\mathrm{s}], \tau_{\mathrm{c}}$ represents the time constant for back flow to the contactor $[\mathrm{s}]$.

when solved, this equation takes the form [7]:

$$
\mathrm{Q}(\mathrm{N})=\mathrm{K}_{1}-\mathrm{K}_{2} \exp \left(-\mathrm{NK}_{3}\right)
$$

where $K_{1}, K_{2}$ and $K_{3}$ are constants and $N$ is the number of subsequent contacts.

Work function theory provides a means of predicting the polarity of the charge on pure surfaces. The magnitude of charge transfer remaining on the surfaces after separation is difficult to predict and thus commercial applications must be based on results from experimentation. It is important to ensure test work is carried out in controlled environmental conditions which exactly reflect those in which the process will be used $[6,7]$. 
For some minerals, despite repeated contacting, the charge magnitude will still be weak. For certain mineral separations this limitation can be overcome by using surface conditioning techniques in advance. This could involve the use of wet or gas phase conditioning. Sodium oleate conditioning has been found [10] to improve the separation of non-conducting material mixtures. The concentrate studied contained cassiterite, scheelite, quartz, mica and tourmaline. Triboelectrification is most frequently used for the separation of two insulators, for example, feldspar and quartz; quartz and apatite mixtures, quartz-calcite mixtures $[11,12]$.

Frictional charge may be applied by a number of different systems:

\section{(i) The Fluidised Bed}

The two materials are dispersed in a fluidised bed and charged to equal and opposite polarities as a result of inter-particle contacts. In practice there is also charge transfer when two particles of the same material contact each other, so this method tends to give poor charge separation between the two materials. This problem can be overcome by reinforcing the action of the bed by an applied electric field. A system of this type is shown in Fig. 1.

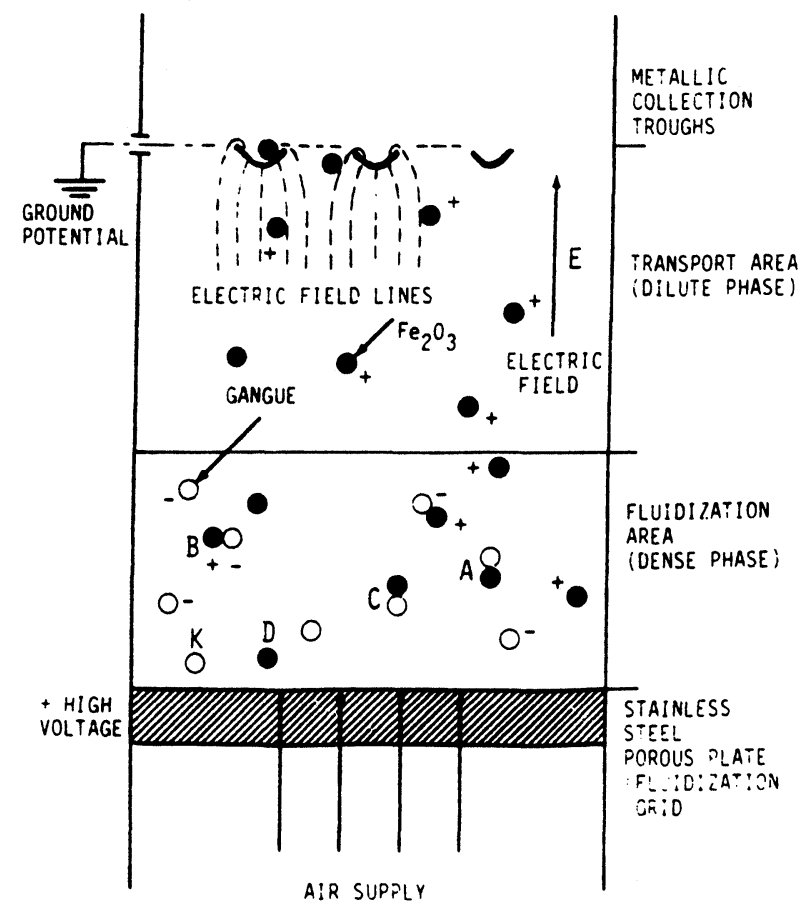

Fig. 1 An electrostatic fluidised-bed separator [6] 


\section{(ii) Rotating Drum}

Two powders can be charged by combining them in a rotating drum. The same problems arise as for the fluidised bed but it has the advantage that the drum can also be used as a kiln when separation is required at elevated temperature [4].

\section{(iii) Vibrating Chute}

When powder falls down a vibrating chute, charge is transferred by contact between the particles and the chute surface. The material of the chute can be selected in order that the two materials charge to opposite polarities. A typical material of construction would be aluminium.

\section{(iv) Air Cyclone}

Cyclones produce high levels of charge and have been tested in a number of different systems. Separation using a cyclone of $10 \mathrm{~cm}$ diameter operating at $0.25 \mathrm{~g} / \mathrm{s}$ has been investigated $[13,14]$ and this work led a triboelectric series for minerals as shown in Table 2.

\section{CORONA CHARGING}

Gases differ from solids and liquids by the manner in which they conduct electricity. In gases at ordinary temperature and pressure, there are neither ions nor electrons and the distance between atoms or molecules is large. This leads to gases behaving as perfect insulators. However, if the potential between electrodes is increased, a stage is reached where the ionisation and conductivity of the gas increase dramatically. This transition, from an insulating to a conducting state, is the electric break-down of the gas and is also referred to as gas discharge. [2]. The ionisation process leads to the emission of light. If using an air medium then a bluish glow is observed around the electrode and this discharge has been named 'corona' [4].

The corona inception voltage can be observed at the point where the current drawn suddenly changes from negligible to approximately $1 \mu \mathrm{A}$. Once corona 


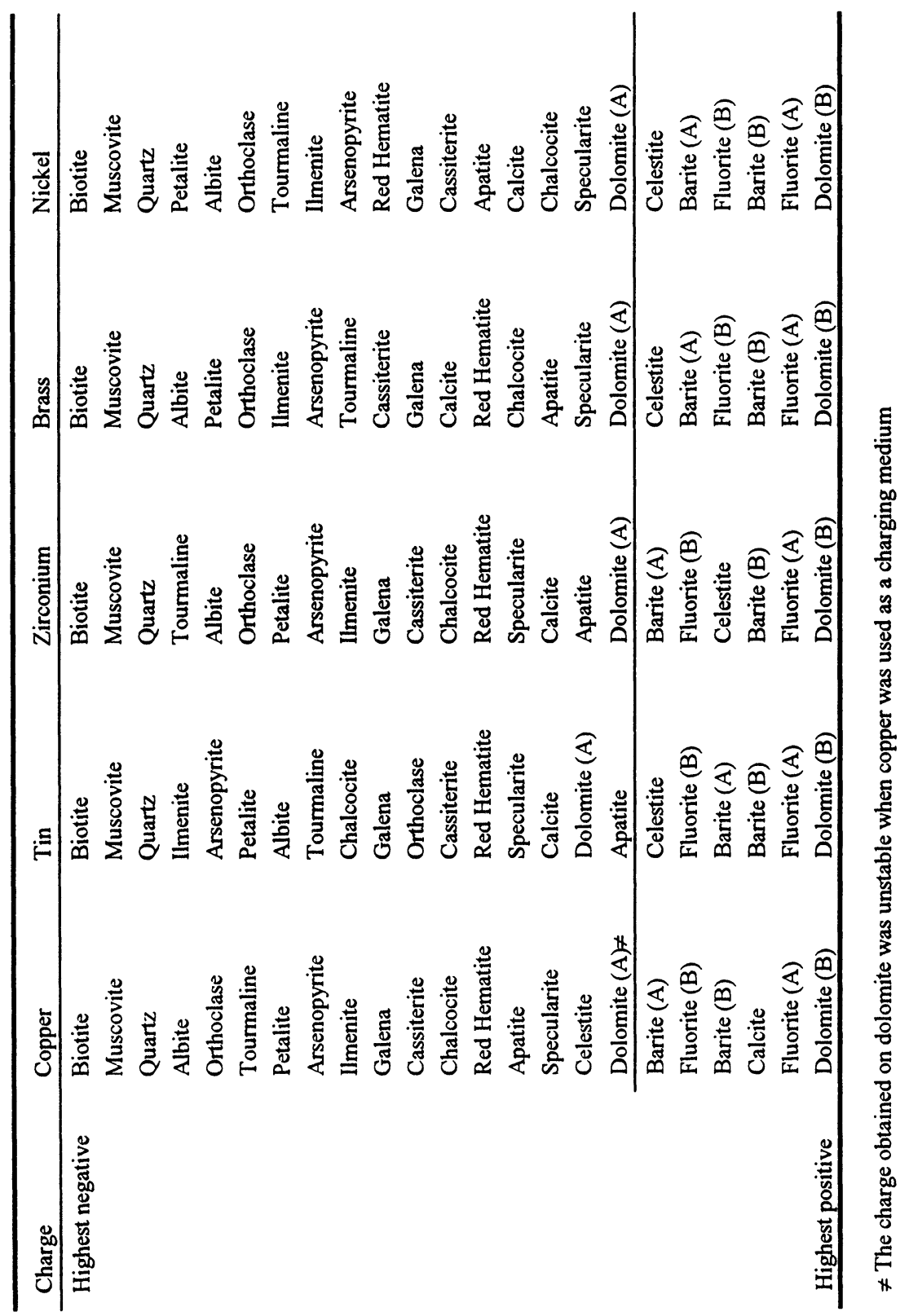

Table 2 The triboelectric series of various minerals on different charging surfaces. A and B designate different samples of the same mineral species [4]. 
discharge commences, the current continues to rise with increasing voltage by the relationship [4]:

$$
\mathrm{I}=\mathrm{KV}\left(\mathrm{V}-\mathrm{V}_{0}\right)
$$

where $V_{0}$ represents the corona inception voltage [V], $I$ is the current $[\mathrm{A}], K$ is a constant which depends on system geometry and $V$ is the voltage [V].

Corona discharge is a low-energy electrical discharge that takes place only in a strong electric field in the vicinity of an electrode of small radius of curvature, and at pressure near to atmospheric. The discharge is self-sustained so no external source of ionisation is needed to maintain the current. The ionisation region is constrained within a small volume close to the discharge electrode, and in the remaining space, ions drift only in the electric field without additional ionisation $[15]$.

Charging mechanisms are not readily predictable. When a surface must be charged in a defined manner, a corona discharge is generally used. This technique generates ions of a single polarity which are capable of charging the surface to a controlled limit. A corona will give a relatively large charge to particles in a short time [4].

The region immediately surrounding a corona electrode leads to particle charging by ion bombardment. Any electron within this region will be accelerated, causing it to gain energy, whereby upon collision with any atom it possesses enough energy to detach an electron. This leads to the production of a positive ion and another electron. This process proceeds until many electrons are accelerated resulting in numerous electron/ion pairs. The movement of gas induced by the repulsion of the ions from the vicinity of a high voltage electrode is referred to as the electric or ion wind. This creates a net motion of gas towards the electric field typically with a speed of the order of $1 \mathrm{~m} / \mathrm{s}[4,16]$.

It is possible to produce both positive and negative corona discharges depending on the polarity of the electrode in use. Both types are used industrially although a negative corona is often preferred since it can produce a more intense corona before the onset of arcing. A negative corona gives rise to a higher corona current than that for a positive corona. The differences between positive and negative corona 
currents, at the same potential, above the onset of corona discharge are ascribable to the difference in ion mobility and electron mobilities [17].

The potential gradient between a wire electrode and the passive electrode (the rotor) is different for negative and positive coronas [18]. For a positive wire there is a gradual potential drop, within this region, while for a negative wire most of the potential drop occurs at the wire.

If a negative, high voltage is applied to an electrode in air, the negative ions entering this region split producing an electron and neutral atom. The electron is accelerated away from the electric field due to the like-charge repulsion, and gains enough energy to ionise atoms upon collision. The electron build-up process continues. At the same time, positive ions become attracted towards the electrode and so are accelerated causing sufficient energy to release secondary electrons on collision. A negative corona is only possible in gases that provide electron attachment $[2,4]$.

If a positive, high voltage is applied, all electrons created in collision leading to ionisation in this region become attracted to the electrode. Positive ions drift away from the electrode but do not gain enough energy to lead to further ionisation. Instead their energy is absorbed by neutral atoms on collision. Most of the current in the space outside the ionisation region is transferred by ions of the same polarity as the electrode and secondary emission is of less importance than for negative coronas. A positive corona is relatively steady and can be produced in almost any gaseous medium $[2,4]$.

Altering polarity is one means of changing the nature of the corona discharge. Other methods include changing electrode design, wave shape, voltage, current or frequency of the power supplying the electrode.

\section{CORONA CHARGING OF PARTICLES}

A particle will polarise when placed within a uniform electric field. This polarisation will cause the particles to attract ions and so a neutral particle will be charged on exposure to a single polarity of ions. 
A conducting sphere in a uniform field is illustrated in Fig. 2. Positive ions will travel along the field lines according to the arrows, negative ions will move in the opposite direction. When a particle is in a corona discharge the ions will follow the field lines and particle charges. As the particles become charged, the field lines modify and ions are collected over a smaller surface area. In a mono-ionised field, charge saturation occurs when the repulsive field caused by the charge of the particle is equal to the attractive field due to the field distortion.

(0)

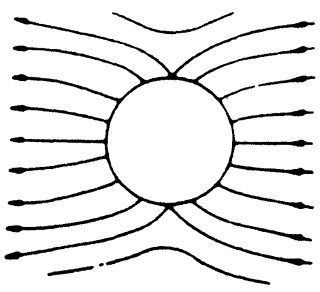

(b)

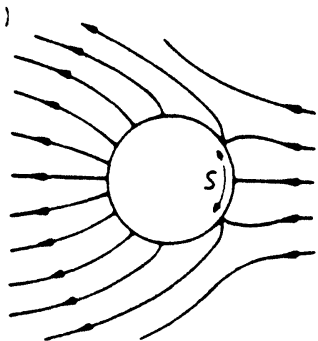

Fig. 2 Electrical field at a conducting sphere in a uniform field: (a) uncharged; (b) with a positive charge [4]

The mechanism of corona charging is still not fully understood. One of the factors that leads to a problem in determination of the exact mechanism is due to the difficulty in measuring the charge accepting properties of fine materials such as powders. For most corona discharges only $0.5 \%$ of the ions produced actually lead to particle charging. The remaining $99.5 \%$ exist as free ions and form the corona wind [19].

Several possibilities exist to describe the exact nature of the processes that will occur when bombarding ions reach the material surface.

- the ion could remain as a stable entity adsorbed on or within the surface layers of the material

- electron transfer might occur to neutralise the ion and so charging the surface states. This would depend on the relative magnitudes of the energy states of the ion and of the surface. Depending on sign, an electron or hole would be introduced into the surface states and the neutralised ion play no further part. 
Whatever the process, the charges initially become located in energy states in the surface region and will need to acquire an activation in order to be freed [20].

\section{CHARGING BY INDUCTION}

When a solid fragment is placed on a grounded conductor, within an electric field, the fragment will quickly acquire a surface charge by induction. The particle is considered to have become polarised whether it be conductive or dielectric [9]. Under such influence, a conductive particle will become an equipotential surface by redistributing the charge almost instantaneously via the grounded rotor.

The particle will acquire a charge opposite to that of the high-voltage electrode generating the electric field [6]. This opposite charging will lead to attraction towards the electrode. A dielectric particle remains polarised because of its inability to effectively redistribute electrons [9]. For this reason, non-conducting particles have no net charge and so are neither attracted or repelled by the field [7].

The charging characteristics will be described by an equation of the form:

$$
\mathrm{Q}=\mathrm{C}_{\mathrm{p}} \mathrm{V}\left[1-\exp \left(-\mathrm{t} / \Omega_{\mathrm{p}} \mathrm{C}_{\mathrm{p}}\right)\right]
$$

where $C_{\mathrm{p}}$ is the capacitance of the particle [F], [C/V], $V$ is the voltage differential $[\mathrm{V}], \Omega_{\mathrm{p}}$ is the particle's equivalent total resistance $[\Omega]$ and $t$ is the time of charging $[\mathrm{s}]$.

\section{REFERENCES}

[1] I. Cakir: Some aspects of the high tension electrical separation of minerals. PhD Thesis, Birmingham University (1979)

[2] A.M. Gaudin: Minerals Sci. Engng. 3, No. 2, (1971), 46-57

[3] British Standards Institutions BS7506, Part 1 (1995) 
[4] J.A. Cross: Electrostatics: Principles, Problems and Applications, Adam Hilger, 1987

[5] E.G. Kelly and D.J. Spottiswood: Introduction to Mineral Processing. John Wiley \& Sons, 1982, p. 291-296

[6] I.I. Inculet: Electrostatic Mineral Separation. Research Studies Press Ltd., 1984

[7] E.G. Kelly and D.J. Spottiswood: Minerals Engineering 2, No. 2 (1989), 193

[8] H.F. Vieweg: J. Phys. Chem. 30 (1926), 865

[9] W.P. Dyrenforth: Electrostatic Separation. Chapter 23: Mineral Processing Design. AIME ed. Muller and Bhappu, 2nd Ed. (1978), p. 479-489

[10] E.E. Rossi del Cerro et al.: Trans. IMM 79, No. 2 (1970), p. C161-163

[11] R.W. Allen: The theory and practice of electrostatic separation. Min. Eng. Thesis, University of Birmingham (1983)

[12] F.S. Knoll and J.B. Taylor: Minerals and Met. Proc. 2 (1985), p. 106-114

[13] M.J. Pearce and M.I. Pope: Powder Tech. 14 (1976), p. 7-15

[14] M.J. Pearce and M.I. Pope: Powder Tech. 17 (1977), p. 83-89

[15] A. Jaworek and A. Krupa: Inst. Phys. Conf. Ser. No. 149, presented 9the Int. Conf. Electrostatics, York, 2-5 April 1995, p. 289-292

[16] R. Robinson: Trans. Am. Inst.Elect. Engrs. 80 (Communications \& Electronics), (1961), 143-150

[17] L.B. Loeb: J. Appl. Phys. 19 (1948), 882

[18] F. Fraas: US Bureau of Mines Bulletin 603 (1962), 72

[19] B.D. Moyle and J.F. Hughes: Inst. Phys. Conf. Ser. No. 66, Session VI, Electrostatics (Oxford), 1983, pp. 155-160

[20] E.A. Baum et al.: J. Phys. D: Appl. Phys. 10 (1977), 487

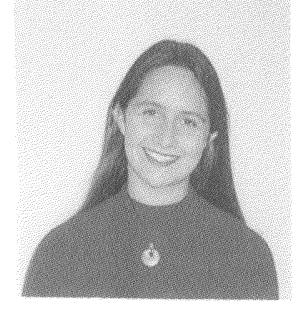

Katharine Lindley was born in 1973 and in 1995 she graduated with a first class honours degree from the School of Chemical Engineering, University of Birmingham, U.K. She is currently studying for a $\mathrm{Ph}$. D. in Mineral Engineering at the University of Birmingham. Her Ph.D. involves the study of electrostatic separation applied to industrial minerals.

Keywords: electrostatic separation, charging mechanisms, triboelectrification 